\title{
THE ANNIHILATOR OF RADICAL POWERS IN THE MODULAR GROUP RING OF A $p$-GROUP
}

\author{
E. T. HILL
}

Abstract. We show that if $N$ is the radical of the group ring and $L$ is the exponent of $N$, then the annihilator of $N^{w}$ is $N^{L-w+1}$. As corollaries we show that the group ring has exactly one ideal of dimension one and if the group is cyclic, then the group ring has exactly one ideal of each dimension.

This paper deals with the group ring of a group of prime power order over the field of integers modulo $p$, where $p$ is the prime dividing the order of the group. This field is written as $K$ and the group ring as $K G$. It is well known that $K G$ is not semisimple; if $N$ is the radical of $K G$ and $N^{L} \neq 0$ while $N^{L+1}=0$, then $L$ is said to be the exponent of $N$. We prove the following result:

Theorem. Let $G$ be a p-group and $K G$ be the group ring of $G$ over $K=G F(p)$, the field with $p$ elements. If $L$ is the exponent of the radical, $N$, of $K G$, then the annihilator of $N^{w}$ is $N^{L-w+1}$.

For $S$ a nonempty subset of $G$, let $S^{+}=\sum_{g_{i} \in S} g_{i}$; in particular, for $H$ a normal subgroup of $G$, let $\left\langle H^{+}\right\rangle$be the ideal in $K G$ generated by $H^{+}$. For $g$ and $h$ in $G$, the following identities are used:

$$
\begin{gathered}
(g-1)^{p-1}=1+g+g^{2}+\cdots+g^{p-1} ; \quad(g-1)^{p}=g^{p}-1 ; \\
(g h-1)=(g-1)(h-1)+(g-1)+(h-1) ;
\end{gathered}
$$

and

$$
(h-1)(g-1)=(g-1)(h-1)+(g h-1)(c-1)+(c-1)
$$

where $c=(h, g)=h^{-1} g^{-1} h g$. The following definitions and theorems are due to Jennings [1].

Let $K_{i}$ be the set of all elements $g_{i}$ in $G$ such that $g_{i} \equiv 1 \bmod N^{i}$.

Theorem 1 [1, Theorem 2.2]. The sets $K_{i}, i=1,2, \cdots$, form $a$ decreasing series of characteristic subgroups of $G$.

This series of subgroups will be referred to as the $K$-series of $G$.

Theorem 2 [1, Theorem 2.3]. The $K$-series of any group $G$ has the following properties:

Presented to the Society, April 18, 1970; received by the editors January 16, 1970. AMS Subject Classifications. Primary 2080; Secondary 1630.

Key Words and Phrases. Modular group ring, radical, annihilator, ideal. 
(1) $\left(K_{i}, K_{j}\right) \subseteq K_{i+j}$;

(2) if $g_{i}$ is in $K_{i}$, then $g_{i}^{p}$ is in $K_{i p}$; and

(3) $K_{i} / K_{2 i}$ is elementary abelian.

By Theorem 2, $K_{i} / K_{i+1}$ is elementary abelian. Let $K_{i} / K_{i+1}$ have order $p^{d_{i}}\left(d_{i}=0\right.$ if $\left.K_{i}=K_{i+1}\right)$, and let $g_{i, 1}, \cdots, g_{i, d_{i}}$ be a complete set of representatives in $G$ of a minimal basis for $K_{i} / K_{i+1}$ (if $K_{i}=K_{i+1}$, let $g_{i, j}=1$ ). In terms of these $g_{i, j}$, for fixed $w$, consider all products of the form $\Pi_{i, j}\left(g_{i, j}-1\right)^{a_{i, j}}$, for $0 \leqq a_{i, j}<p$, with $\sum_{i, j}\left(i a_{i, j}\right)=w$; the summation extending over the same $i$ and $j$ as in the product. Those factors which are present in the product are in the natural order of increasing $i$ and $j$. Call these various distinct products $N_{1}^{w}, N_{2}^{w}, \cdots, N_{t_{w}}^{w}$.

Theorem 3 [1, Theorem 3.6]. The elements $N_{i}^{w}$ for fixed $w$ form a basis for $N^{w}$ modulo $N^{w+1}$. The number $t_{w}$ of these elements is the dimension of $N^{w} / N^{w+1}$.

Theorem 4 [1, Theorem 3.7]. The dimension $t_{w}$ of $N^{w} / N^{w+1}$ is equal to the coefficient of $x^{w}$ in the expansion of $\left(1+x+x^{2}+\cdots+x^{p-1}\right)^{d_{1}} \cdots\left(1+x^{i}+x^{2 i}+\cdots+x^{i(p-1)}\right)^{d_{i}} \cdots$ and the exponent $L$ of $N$ is equal to $\sum_{i} i d_{i}(p-1)$.

The $M$-series for $G$ is defined as follows: $M_{1}=G$; for $i>1$, $M_{i}=\left\langle\left(M_{i-1}, G\right), M_{(i / p)}^{p}\right\rangle$ where $(i / p)$ is the least integer not greater than $i / p$ and $M_{k}^{p}$ is the set of all pth powers of elements of $M_{k}$.

Theorem 5 [1, Theorem 5.5]. The $M$-series and the $K$-series are identical.

The notation, definitions, and results of Jennings stated above will be used throughout this paper.

LemMA 6. If $G_{1} \supset G_{2} \supset \cdots \supset G_{m}=1$ is a composition series in $G$ and $g_{i}$ is a representative in $G$ of a minimal basis for $G_{i} / G_{i+1}$, then $\left(g_{i}-1\right)^{p-1} G_{1+1}^{+}=G_{i}^{+}$.

Proof. The cosets of $G_{i} / G_{i+1}$ will be $G_{i+1}, g_{i} G_{i+1}, \cdots, g_{i}^{p-1} G_{i+1}$. Hence, $\left(g_{i}-1\right)^{p-1} G_{i+1}^{+}=\left(1+g_{i}+g_{i}^{2}+\cdots+g_{i}^{p-1}\right) G_{i+1}^{+}=G_{i}^{+}$.

THEOREM 7. Let $x=\prod_{i \geqq m}\left(g_{i, j}-1\right)^{p-1}$ for some fixed positive integer $m$, the factors being in the natural order of increasing $i$ and $j$, and the product taken over all $\left(g_{i, j}-1\right)$ with $d_{i} \neq 0$. If $m$ is such that $d_{j} \neq 0$ for some $j \geqq m$, then $x=M_{m}^{+}$. 
Proof. The proof is inductive. Let $k$ be the largest integer such that $d_{k} \neq 0$. By Theorems 2 and $5, M_{k}$ is elementary abelian. Notice that $M_{k}=\left\langle g_{k, 1}, g_{k, 2}, \cdots, g_{k, d_{k}}\right\rangle \supset\left\langle g_{k, 2}, \cdots, g_{k, d_{k}}\right\rangle \supset \cdots \supset\left\langle g_{k, d_{k}}\right\rangle \supset 1$ is a composition series, so that Lemma 6 applies and $M_{\boldsymbol{k}}^{+}$ $=\prod\left(g_{k, j}-1\right)^{p-1}$. Hence the theorem is proved for $m=k$.

Suppose the theorem is true for $m=r+1$. If $d_{r}=0$, then $M_{r}=M_{r+1}$ and there is nothing to prove. If $d_{r} \neq 0$, then $x=\prod_{i \geqq r}\left(g_{i, j}-1\right)^{p-1}$ $=\prod\left(g_{r, j}-1\right) M_{r+1}^{+}$. By Theorems 2 and $5 ; M_{r} / M_{r+1}$ is elementary abelian and, as above, $x=M_{r}^{+}$. Hence the theorem is true for all applicable $m$.

In particular, $x$ of this form for $m=1$ implies that $x=M_{1}^{+}=G^{+}$. Therefore, $N^{L}=\left\langle G^{+}\right\rangle$.

Define a function $f$ on the $N_{k}^{w}, f\left(N_{k}^{w}\right)$ a product of powers of the $\left(g_{i, j}-1\right)$ such that: if $d_{i}=0$, then the term $\left(g_{i, j}-1\right)$ does not appear in $f\left(N_{k}^{w}\right)$; if $d_{i} \neq 0$, then $f\left(N_{k}^{w}\right)$ has a factor $\left(g_{i, j}-1\right)^{b_{i, j}}$ with $b_{i, j}$ $=(p-1)-a_{i, j}$ where $a_{i, j}$ is the exponent on the corresponding term in $N_{k}^{w}$. The factors are arranged in the natural order of increasing $i$ and $j$.

Notice that $0 \leqq b_{i, j}<p$ and the factors are in the proper order so that $f\left(N_{\boldsymbol{k}}^{w}\right)$ is some $N_{r}^{m}$, a basis element for $N^{m} / N^{m+1}$. Since $m=\sum_{i, j} i b_{i, j}=\sum_{i, j} i\left((p-1)-a_{i, j}\right)$ and for each $i$ summing over $j$ yields $d_{j}$ factors of $(p-1), m=L-w$ by Theorem 4 . Hence, $f\left(N_{\boldsymbol{k}}^{w}\right)$ is some $N_{r}^{L-w}$. Clearly, $f\left(f\left(N_{k}^{w}\right)\right)=N_{k}^{w}$.

Lemma 8. For $f$ defined above, $f\left(N_{k}^{w}\right) \cdot N_{k}^{w}=G^{+}$.

Proof. Let $t$ be an integer such that $M_{t}=1$. Then for $m=t$ the product has the form

$$
f\left(N_{k}^{w}\right) \cdot N_{k}^{w}=\pi_{1} \pi_{2} M_{m}^{+}
$$

where $\pi_{1}$ is a product of $\left(g_{i, j}-1\right)$ from $f\left(N_{\boldsymbol{k}}^{w}\right)$ with $i<m$ and $\pi_{2}$ is a similar product from $N_{k}^{w}$.

Suppose the product has the form (1) for $m=r+1$. If $d_{r}=0$, then $M_{r}=M_{r+1}$ and the product has form (1) for $m=r$. If $d_{r} \neq 0$, write

$$
f\left(N_{k}^{w}\right) \cdot N_{k}^{w}=\pi_{1}^{\prime}\left(g_{r, j}-1\right)\left(g_{s, i}-1\right) \pi_{2}^{\prime} M_{r+1}^{+}
$$

and let $c=\left(g_{r, j}, g_{s, i}\right)$. Then

$$
\left(g_{r, j}-1\right)\left(g_{s, i}-1\right)=\left(g_{s, i}-1\right)\left(g_{r, j}-1\right)+\left(g_{s, i} g_{r, j}\right)(c-1),
$$

so that

$$
\begin{aligned}
f\left(N_{j}^{w}\right) \cdot N_{j}^{w}= & \pi_{1}^{\prime}\left(g_{s, i}-1\right)\left(g_{r, j}-1\right) \pi_{2}^{\prime} M_{r+1}^{+} \\
& +\pi_{1}^{\prime}\left(g_{s, i} g_{r, j}\right)(c-1) \pi_{2}^{\prime} M_{r+1}^{+} .
\end{aligned}
$$


By Theorems 2 and 5,c is in $M_{r+1}$; the second term in the sum has factors of $(c-1)$ and $M_{r+1}^{+}$, so this term is 0 . Hence, the $\left(g_{r, j}-1\right)$ terms commute to their natural position in the product. Since the exponent on the $\left(g_{r, j}-1\right)$ term in the product is $a_{r, j}+b_{r, j}=p-1$, the product has form (1) for $m=r$ by Theorem 7 . Therefore, the product has form (1) for $m=1$ and the lemma is proved.

Let $a_{i, j}$ be the exponent on the $\left(g_{i, j}-1\right)$ term in $N_{\boldsymbol{k}}^{w}$ and let $b_{i, j}$ be the exponent on this term in $N_{m}^{w}$; if the $\left(g_{i, j}-1\right)$ term does not occur in $N_{k}^{w}$, let $a_{i, j}=0$ with similar convention in $N_{m}^{w}$. Define $N_{\mathfrak{k}}^{w} \ll N_{m}^{w}$ if there are positive integers $s$ and $t$ such that $a_{s, t}<b_{8, t}$ and $a_{i, j}=b_{i, j}$ for all $i$ and $j$ such that $i>s$ or $i=s$ and $j>t$. That is, terms to the right of $\left(g_{8, t}-1\right)$ have the same exponents and $a_{s, t}<b_{s, t}$. Clearly any two distinct basis elements are comparable and this ordering is transitive.

LeMma 9. If $f\left(N_{\mathbf{k}}^{w}\right) \ll N_{m}^{L-w}$, then $N_{m}^{L-w} \cdot N_{\mathfrak{k}}^{w}=0$.

Proof. Let $s$ and $t$ be as in the definition of $f\left(N_{\boldsymbol{k}}^{w}\right) \ll N_{m}^{L-w}$. As in Lemma 8 , the terms $\left(g_{i, j}-1\right)$ with $i \geqq s+1$ commute to their natural position so the product has the form

$$
N_{m}^{L-w} \cdot N_{k}^{w}=\pi_{1} \pi_{2} M_{8+1}^{+} .
$$

The terms $\left(g_{s, j}-1\right)$ also commute to their natural position by the proof of Lemma 8 . The exponent on the $\left(g_{s, t}-1\right)$ term in the product is $a_{s, t}+b_{s, t}$ where $a_{s, t}$ is the exponent from the term in $N_{m}^{L-w}$ and $b_{s, t}$ is the exponent in $N_{w}^{k}$. The exponent of $\left(g_{s, t}-1\right)$ in $f\left(N_{k}^{w}\right)$ is $(p-1)-b_{s, t}$ and $a_{s, t}>(p-1)-b_{s, t}$ so that $a_{s, t}+b_{s, t}>(p-1)$. Let $a_{s, t}+b_{s, t}=p+c_{s, t}$. Then $\left(g_{s, t}-1\right)^{a_{s, t}+b_{s, t}}=\left(g_{s, t}^{p}-1\right)\left(g_{s, t}-1\right)^{c_{s, t}}$. By Theorem $2, g_{s, t}^{p}$ is in $M_{s+1}$. The product has factors of $\left(g_{s, t}^{p}-1\right)$ and $M_{s+1}^{+}$; therefore, the product is 0 and the lemma is proved.

For $I$ an ideal in $K G$, let $0: I$ be the left annihilator of $I$ and $A(I)$ be the annihilator (two-sided) of $I$.

Lemma 10. 0: $N^{w}=N^{L-w+1}$.

Proof. Clearly $0: N^{L}=N$; suppose that $0: N^{w+1}=N^{L-w}$. Then $N^{w+1} \subseteq N^{w}$ implies $0: N^{w} \subseteq 0: N^{w+1}=N^{L-w}$ so that $N^{L-w+1} \subseteq 0: N^{w}$ $\subseteq N^{L-w}$. The lemma will be proved if $x$ is a linear combination of the $N_{i}^{L-w}$ and $x$ is in $0: N^{w}$ implies $x=0$.

For $f$ defined above, if $N_{k}^{w} \ll N_{m}^{w}$, then clearly $f\left(N_{m}^{w}\right) \ll f\left(N_{k}^{w}\right)$. Let $N_{1}^{w}$ be the least $N_{i}^{w}$ under $\ll, N_{2}^{w}$ the second smallest and so on; let $N_{m}^{w}$ be the largest. Order the $N_{i}^{L-w}$ in the same manner. Then $f\left(N_{m}^{w}\right)=N_{1}^{L-w}$ and for $m>k \geqq 0, f\left(N_{m-\mathbf{k}}^{w}\right)=N_{\boldsymbol{k}+1}^{L-w}$ since $f$ reverses the order. 
Let $x=\sum a_{i} N_{i}^{L-w}$ be a linear combination of the $N_{i}^{L-w}$ ordered as above and suppose that $x$ is in $0: N^{w}$. By Lemmas 8 and $9, x \cdot N_{m}^{w}=a_{1} G^{+}$ since $N_{1}^{L-w} \cdot N_{m}^{w}=f\left(N_{m}^{w}\right) \cdot N_{m}^{w}=G^{+}$and $N_{i}^{L-w} \gg f\left(N_{m}^{w}\right)$ implies $N_{i}^{L-w} \cdot N_{m}^{w}$ $=0$. Hence, if $x$ is in $0: N^{w}$, then $a_{1}=0$. Suppose that $x$ in $0: N^{w}$ implies $a_{i}=0$ for $i \leqq k$. As above, $x \cdot N_{m-\mathbf{k}}^{v}=a_{k+1} G^{+}$since $a_{i}=0$ for $i \leqq k$, $N_{k+1}^{L-w} \cdot N_{m-k}^{w}=f\left(N_{m-k}^{w}\right) \cdot N_{m-k}^{w}=G^{+}, \quad$ and $N_{i}^{L-w} \cdot N_{m-k}^{w}=0$ for $N_{i}^{L-w}$ $\gg f\left(N_{m-k}^{w}\right)$. Therefore, $x$ in $0: N^{w}$ implies $a_{k+1}=0$ so that $a_{i}=0$ for $1 \leqq i \leqq m$ and $x=0$.

The proof of the main theorem follows immediately.

Theorem 11. $A\left(N^{w}\right)=N^{L-w+1}$.

Proof. By Lemma 10, $N^{L-w+1}=0: N^{w} \supseteq A\left(N^{w}\right) \supseteq N^{L-w+1}$.

Corollary 12. If $I$ is an ideal of dimension $w$ and $w \leqq L$, then $I \subseteq N^{L-w+1}$.

Proof. For $I$ as above. $I \cdot N^{w}=N^{w} \cdot I=0$ so that $I \subseteq A\left(N^{w}\right)=N^{L-w+1}$.

Corollary 13. $K G$ has exactly one ideal of dimension one; this ideal is $\left\langle G^{+}\right\rangle$.

Proof. If $I$ has dimension one, then $I \subseteq N^{L}=\left\langle G^{+}\right\rangle$; since $\left\langle G^{+}\right\rangle$has dimension one, $I=\left\langle G^{+}\right\rangle$.

Corollary 14. $G$ cyclic implies $K G$ has exactly one ideal of each dimension.

Proof. If $G$ is cyclic of order $p^{n}$, then $N^{p^{n}}=0$ and $N^{p^{n}-1} \neq 0$. Hence $(g-1)^{w}$ is a basis for $N^{w} / N^{w+1}$ where $g$ generates $G$. Also, $N^{L-w+1}$ has dimension $w$. If $I$ is an ideal of dimension $w$, then $I \cdot(g-1)^{w}=0$; therefore, $I \subseteq 0: N^{w}=N^{L-w+1}$. Since $I$ and $N^{L-w+1}$ have the same dimension, they are equal.

\section{REFERENCES}

1. S. A. Jennings, The structure of the group ring of a p-group over a modular field, Trans. Amer. Math. Soc. 50 (1941), 175-185. MR 3, 34.

Cornell College, Mount Vernon, Iowa, 52314 